\title{
Construction of Ecological Chain of Urban Sustainable Education under the Background of Informatization -Taking "Knowledge- Practice- Parks" volunteer service team as an example
}

\author{
Wang Kewei ${ }^{1, a,{ }^{*} \text {, Zhang Zhiheng }}{ }^{2, b}$, Wang Yongli ${ }^{3, c}$ and Yu Xintong ${ }^{4, d}$ \\ ${ }^{1}$ Central China Normal University, Wu Han, China \\ ${ }^{2}$ Central China Normal University, Wu Han, China \\ ${ }^{3}$ Central China Normal University, Wu Han, China \\ ${ }^{4}$ Central China Normal University, Wu Han, China \\ awkw1995@163.com, b495404121@qq.com, ‘wyli1995@163.com, d1021459631@qq.com \\ *Wang Kewei
}

Keywords: City, Sustainable education, Ecological chain.

Abstract: In the age of information, the sustainable development of education is confronted by the incomplete education objects, the weak organization, the out of life contents and other difficulties. Based on the practical experience of "Knowledge. Practice- Parks" volunteer service team in Wuhan, this thesis puts forward the concept of " Ecological Chain of Urban Sustainable Education ", combining with the new environment of large community and learning city, then tables some applicable proposals from the perspectives of organizational pattern, resource development, innovation in carriers and so on.

\author{
信息化背景下城市可持续发展教育 \\ 的生态链构建 \\ 一以 “知行园” 志愿服务队为例 \\ 王辑玮 $1, \mathrm{a},{ }^{*}$, 张志恒 ${ }^{2, b}$, 王永立 ${ }^{3, c}$, 于欣粀 $4, \mathrm{~d}$ \\ 1华中师范大学, 武汉, 中国 \\ 2华中师范大学, 武汉, 中国 \\ 3华中师范大学, 武汉, 中国 \\ 4华中师范大学, 武汉, 中国
}

awkw1995@163.com, b495404121@qq.com, ‘wyli1995@163.com, d1021459631@qq.com

*王辑玮

关键词：城市; 可持续发展教育; 生态链

中文摘要. 在信息化时代中, 可持续发展教育面临教育对象不全面、实施组织薄弱化、内容 脱离生活等困境。本文从武汉市知行园志愿服务队的实践经验出发, 结合大社区、学习型城 市的新环境, 提出 “可持续发展教育生态链” 的概念, 从组织范式、资源开发、载体创新等 角度提出应用建议。 


\section{1. 引言}

自2005 年, 联合国启动可持续发展教育十年活动至今, 与可持续发展有关的教育活动已 经在全球范围铺开。与传统环境教育的理念不同, 可持续发展教育主张以可持续发展的思想 为核心, 从系统的角度来分析环境问题。将环境问题置于经济、社会、文化大环境中探讨原 因与对策。[1]在中国, 可持续发展教育的历史并不悠久, 目前主要实施于学校及城市社区。 “知行园”志愿服务活动就是一个带有明显中国特色的可持续发展教育案例。它由华中师范大 学学生自主发起, 围绕可持续发展这一核心内容, 将课程学习、学术研究和社会服务融为一 体, 面向广大市民进行宣讲服务。目前已经在武汉市 32 个公园设立常规活动点, 直接受益人 数超过 5000 人。本文以“知行园”志愿服务团队为例, 结合实证调查结果, 探讨城市可持续发 展教育的创新策略。

\section{2. 信息化时代城市可持续发展教育面临的困境}

近年来信息技术的迅猛发展, 很大程度上改变了城市原有的功能结构, 间接影响了城市 公共教育的实效性。我们针对可持续发展教育的推进现状, 在武汉市选取了以中山公园为代 表的 4 个市民集结地作为样本, 进行实证调查。共回收问卷 268 份, 访谈 24 人（其中 15 人为社 区工作人员），现将发现的负面影响归纳如下:

\section{1 主体狭义化: 可持续发展教育的对象不全面}

可持续发展教育在实施过程中，应重视培养各年龄阶段的市民对环境的敏感度和获得有 关知识、解决问题的技能, 尤其要重视培养成人对环境的敏感度与社会责任感。[2]但当前大 多数教育机构包括社区、文化馆等在组织可持续发展教育活动时常常把青少年群体列为主要 对象。诚然青少年是社会的新希望、新力量, 但是成年人的主体地位是不可忽视的。据问卷 调查显示, 有 $82.5 \%$ 的人表示从未接受过可持续发展教育或其他类似环境教育, 其中年满 30 周岁的成年人占比超过 $70 \%$ 。问卷调查所反映的情况与访谈结果基本一致，15位社区工作者 中有 12 人表示近两年来没有开展过专门针对成年人的环境教育。大多数环保宣传是通过分发 传单, 张贴海报的形式进行的。可见狭义化的主体观直接导致可持续发展教育的对象不够全 面。

\section{2 组织薄弱化: 可持续发展教育的氛围不活跃}

根据联合国教科文组织的定义，社区教育指代以提高社区住民生活质量为目的一系列教 育活动。在城市可持续发展教育这一领域中, 社区教育的份额是不可忽视的。它与传统学校 的环境教育相比具有辐射面广、影响力大的特征。

随着城市结构进一步转型, 高度合一的由政府主导的城市生态正在发生改变。以社区为 代表的非官方力量被不断强化。但是近年来, 网络技术的进步直接推动了虚拟社区在居民生 活中的渗透。当今意义上的城市社区不再仅仅是一个单纯的地理概念, 社会结构渐渐被网络 重组。过去围绕地理性社区所开展的可持续发展教育面临重大挑战, 从参与率、活动效果到 意见反馈均出现倒退。据调查显示, 经常参加社区活动 (不低于 1 个月 2 次) 的居民只有 $15.3 \%$, 且平均年龄超过 65 岁, 偶尔参加的（不低于 3 个月次）占总数的 $23.1 \%$ 。此处对于社区活动的 界定主要指由居委会或楼管会发起的活动, 不包括广场舞等自发娱乐行为。居民积极性不高, 社区号召力不强是受访对象认为最严重的问题。其次是基础设施不完善, 缺乏合适的教学场 地。

\section{3 课程理论化: 可持续发展教育的推行脱离市民需求}

在课程设置方面, 现行的城市可持续发展教育缺乏分层细致的社会性读本或教材。部分 教育者在实践中参考的是高校人才培养方案以及中学课程。从理论的前沿性和科学性来说是 
没有问题的。但是对市民而言，尤其是工作稳定、年龄偏大的中年群体，他们更关心知识的 实用性。我们摘选了3份过去用于环保宣传的传单、2份社区活动的讲稿（节选）, 借此调查 市民对上述内容的主观感受。有 $62.7 \%$ 的人认为相关内容存在脱离生活的倾向; $24.6 \%$ 的人觉 得文本过于单调，导致兴趣降低； $18.7 \%$ 表示对内容基本满意。另有 $6 \%$ 的人选择“没有明晰的 态度”。总体来说，当前可持续发展教育的内容设计一定程度上不符合市民生活的实际需要。

\section{3. 新理念、新环境: 可持续发展教育的时代机遇}

虽然可持续发展教育存在上述困境，但是从大环境来看，“大社区”服务、“学习型”城市、 “网格化”管理等新理念、新环境的出现为可持续发展教育提供了重要的创新契机。探索“大学 (University）+社会（Society）+网络（Network）”（简称“USN”）立体化教育模式变成了一 种可能。

\section{1 大社区理念}

大社区理念的出现是为了进一步推动社区自治, 改变“强政府, 弱社会”的局面。它主张 培育民间组织, 统筹各方资源, 转变管理方式。[3]受到大社区理念的影响, 民间组织、基层 居委会与政府之间不再是单纯管理与被管理的关系, 而是演变为一个互为补充、相互融合的 联合体。近年来, 民间组织的数量不断增加, 开始承担越来越重要的社会职责。社区教育也 渐渐成为城市可持续发展教育的核心组成部分。

\section{2 学习型城市}

在我国，学习型社区的创建刚刚兴起不久，还是一个新生物。在社区营造“全员、全程、 全方位”的学习氛围, 形成文明、和谐、健康的人文环境, 是可持续发展教育活动在城市落地、 扎根的重要保证, 也是大范围普及可持续发展知识的一个契机。以“知行园”团队所在的武汉 为例。武汉是中国重要的中心城市。目前在武汉的市辖区中, 已有青山区、江岸区、新洲区 等4个区被确定为全国社区教育实验区。[4]实施名牌教育战略, 贯彻终生教育的理念, 充分 利用博物馆、活动室、公园等公共场所开展教育活动, 形成了百步亭社区等一批学习型社区 典范。为可持续发展教育与市民生活的衔接提供了可能性。

\section{3 微观污染普遍}

自从PM2.5被纳入《环境空气质量标准》, “穹顶之下”等一系列环保纪录片在引起社会广 泛关注的同时, 也一定程度上增加了公众对于城市污染的危害认知。如何从生活源头做起减 少污染源, 如何在生活细节中避免受污染, 成为大家普遍关心的一个话题。相关媒体机构、 教育组织借此机会, 增加环保知识、可持续发展理念传播的广度和深度, 从过去零散、自发 地宣传演变成今天自觉、有序地传播。这也为城市可持续发展教育提供了广大的受众市场和 良好的创新空间。

\section{4. 城市可持续发展教育的个体经验: 以知行园志愿服务团队为例}

“知行园”志愿服务队成立于2016年4月。它的诞生与上述的时代背景紧密相连。与普通的 学生实践队、爱心社团不同, “知行园”志愿服务队将学术科研与志愿服务结合在一起, 贯彻 “USN”思想, 把高校内最新的学术成果、可持续发展理念带入社区, 带进网络, 充分利用社 会资源。现已在武汉市中山公园创设“大学生“公园大课堂”志愿服务基地”。它的运行模式、工 作方法可以有效地解决上述在实证调查中发现的问题困境，利用信息化，发挥时代优势。 


\section{1 创新课程内容, 迎合市民兴趣及需求}

“知行园”志愿服务队重要的常规活动包括“公园大课堂”与“乐吧学堂”，即立足于公园、 景区等公共设施, 进行环境保护的主题宣讲。对可持续发展教育的内容进行合理地分解、创 新、延伸, 是开展宣讲活动的第一步。为此服务队综合《关于推进社区教育工作的若干意见》 等政策文件, 对课程内容进行重新设计, 在讲解环节之外还加入了自然体验、情感素质、户 外运动等趣味形式。例如在以“垃圾分类”为主题的教育活动时, 鼓励市民带着孩子一同报名, 采用了“创意谜语竞猜、垃圾桶化妆秀、快问快答” 等环节, 既保证了活动的亲子性, 吸引市 民参与，同时注重行为的引导。

\section{2 采用小组化管理, 深入社区网格}

知行园志愿服务团队目前依托所在高校（华中师范大学），已经与市政部门（武汉市园 林局）、社会媒体（长江日报）及其他公园、社区建立了长效联系机制，努力改善可持续发 展教育组织力薄弱的问题。在教育对象的选择上, 团队将青少年群体和30-50岁的中青年群体 作为主要对象。但没有设置明确的年龄限制。从实际情况来看, 以家庭为单位来参加公益活 动的居民数量是最多的。除了创意型的活动课程之外, 进社区宣讲、调研也是知行园志愿服 务团队的重要内容之一。为了便于分散宣讲和走访调研, 团队采用小组化工作的方法, 划分 为学术理论小组（负责内容产出）、宣传活动小组（负责对接社区和前期预热）以及若干个 调研讲解小组, 根据每周的主题和社区具体环境再作实地调整, 努力提高了对社区网格的辐 射力度。

\section{3 打造新媒体矩阵, 适应信息化潮流}

为了适应信息化, “知行园”团队融合矩阵思想, 依托社会媒体及学校校媒, 例如长江日 报、武汉公园客、CCNU马院助手等, 定期发布活动预告、报名信息、生活小常识以及可持 续发展理念。一方面, 市民可以通过微信公众号了解活动预告, 参加线上报名, 并及时进行 意见反馈; 另一方面, 团队可以利用校园媒体, 传播公益理念, 扩大自身知名度, 吸引更多 志同道合的学生加入其中, 确保团队的长效运行。网络新媒体实际上在这一过程中扮演的是 一个串联社会、高校等场域的信息桥梁。

\section{5. 基于实践经验的模式创新：打造可持续发展教育的生态链}

“知行园”志愿服务团队的实践为改善当前信息化背景下城市可持续发展教育的现状提供 了现实经验。将生态链思想引入可持续发展教育领域, 有助于进一步凝练实践经验, 为今后 的推广创新提供借鉴。

\section{1 可持续发展教育生态链的模式原理}

在自然界，任何生物个体都无法单独长期存在，只有形成一个群体后才能繁衍后代。[5] 一般而言, 一个生态群落里具备生产者、消费者、分解者三种不同角色的生物才能够维持整 个群落的可持续循环发展。结合“知行园”志愿服务团队的创新实践, 参考“大学 (University) +社会 (Society) +网络（Network）”的思维，城市可持续发展教育系统对应于自然生态系统 中的三要素, 也有类似的角色分工。具体分析如下:

生产者是生态系统中最基础的成分, 它们能够为其他一切异养生物提供生存资源。[6]可 持续发展教育生态链中, 生产者具体指代学校 (含高校和中小学) 、科研机构、公益组织等, 他们是可持续发展教育活动的组织者、创造者、参与者。承担着发掘教育内容, 创新方式载 体的重要任务。

消费者在自然生态系统中主要是各种动物, 它们直接或间接地依赖于生产者制造的有机 物, 对初级生产物有着加工、再生产的作用。而在可持续发展教育中, 涵盖了多个消费者群 
体, 按照他们的需求不同, 可以分为三级消费者。即生活消费者（居民）、学习消费者（学 生）、发展消费者（企业）。它们的需求呈现多层次多样的特点。

分解者在自然生态系统中主要以细菌和真菌为主, 它们把复杂的有机物分解还原为简单 的无机物, 最终以无机物的形式回归到环境中。我们认为可持续发展教育领域的还原者应为 城市信息综合处理系统。它的职能类似于目前政府机构中存在的数据统筹局, 可以由政府管 理中心牵头, 在城市信息处理中心的基础上进行构设。具体承担信息分析、活动反馈的职能, 肩负改善基础条件、吸引受众的责任。

\section{2 可持续发展教育生态链的模式概述}

一个良性运行的可持续发觉教育生态链除了存在生产者、消费者、分解者和环境条件外, 与自然生态系统一样, 也要有信息、能量、价值等的流动。

下图为基于生态系统原理所构建的可持续发展教育生态链模型。该模型中，三级消费者 除了消费生产者所生产的资源之外, 彼此之间还会有物质流动, 存在反向消费。在模型运行 过程中, 多主体的利益相关者通过社会与经济复合的生态系统形成了复杂的互动关系与影响 机制, 生产者、消费者、分解者三者密不可分。其中处于中枢地位的分解者是生态链的关键 部门。在信息化时代中, 他们除了要履行常规的管理、服务职能之外, 还要运用数字化、云 平台等手段, 整合可持续发展教育的各种资源, 统筹规划, 从需求出发, 将可持续发展教育 工作推到实处。

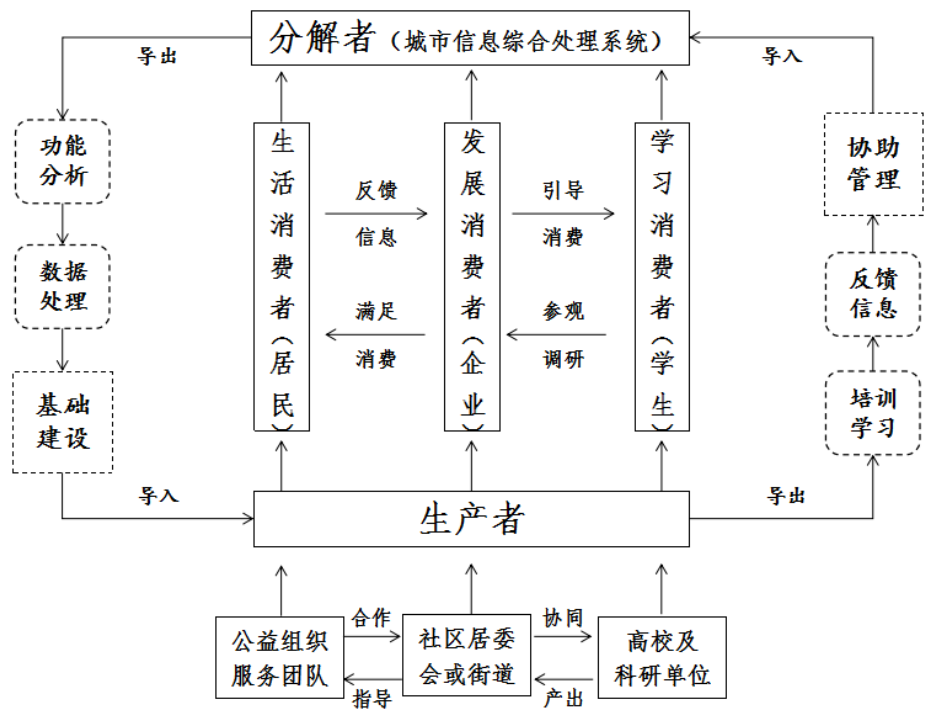

图1 可持续发展教育生态链模式图

\section{6. 可持续发展教育生态链的应用要点}

\section{1 组织关系：遵循“多中心型同心圆”的范式}

在生态链模型中，学校、公益组织、社区、政府不在是过去管理与被管理或完全被割裂 的关系, 他们之间的互动反馈、双向交流如今显得更加重要。城市信息综合处理系统作为分 解者, 处于中心地位。与自然世界中的生态链不同, 在可持续发展教育生态链模式中, 分解 者的中心地位是常态化的, 把握导向; 但是学校、社区等其他社会主体同样可以是一个节点, 也可以成为一个动态具有流动性的活动中心。他们的活动中心地位是根据实际情况的不同而 不断更迭变化的。所以他们之间的关系不同于以往普通的“同心圆”, 这是一种具有多个中心 的新型“同心圆”。它存在一定的群体聚集特征, 呈现一种更为自由和自主的形态。共同参与、 权级平等的交流组织结构初步成型。 


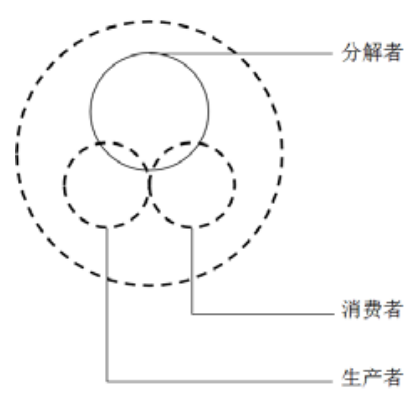

图2 多中心同心圆组织范式

\section{2 资源开发: 教育内容与兴趣事物的接洽设计}

在落实生态链模式过程中，要认清不同主体间的差异，充分发挥各自优势，做好协同交 互。例如可持续发展教育的内容, 需要做到与教育对象的兴趣事物衔接。作为生产者, 科研 机构和高校的主要任务是负责内容产出, 精选最新的科技成果和环保领域的研究成果, 丰富 教学宣讲的内容; 社区及公益组织由于贴近居民生活, 由他们对产出内容进行改造, 从流行 文化和市民心理出发, 用喜闻乐见的生活趣事或真人真事勾起情感共鸣, 让教育内容趋向生 活化、现实化，唤醒社区““沉睡”的精力、时间、财富和智慧。

\section{3 载体创新: 活动方式与互联网的并用设计}

信息化是不可逆的时代背景。因此, 可持续发展教育生态链模式要将运用网络技术和使 用新媒体平台作为重要手段进行开发和运用, 配合已有的数据分析平台, 进行数据挖掘、个 性化推存。从线上发力, 打通线下的实体活动。尤其是分解者作用的发挥离不开 $\mathrm{O} 2 \mathrm{O}$ 思想 （online to offline）, 线上活动与线下活动彼此不是独立各自进行的关系。线上活动应该是线 下活动的补充和拓展, 它需要发挥它的渠道作用, 但是它并不是教育活动的主体, 不能取代 线下活动的聚心、凝力的作用。因此在实践过程中需要重点关注线下活动与移动互联网的并 用设计，减少“单向割裂”的现象。

综上所述, 可持续发展教育生态链模型的出发点在于协同互利, 积极发挥三种角色的作 用, 最终实现惠及城市公共教育、改进可持续发展教育的双重发展。事实上, 生态链模型并 不是对可持续发展教育的工作模式提出变革性的想法, 它更多是针对具体工作的结构性改善 建议。希望通过一种模式上的创新对可持续发展教育进行资源整合、形式再造、提高相关活 动的质量和文化内涵。

\section{References}

[1] Min Wang, Dongying Wei, Zhiling Huo, Environmental education and sustainable development education, JOURNAL OF BEIJING NORMAL UNIVERSITY (SOCIAL SCIENCES), vol.3, pp.131-136, 2006.

[2] Xiaowei Fan, Research on sustainable development education of Shanghai citizens, master's thesis of East China Normal University, 2015.

[3] Chong Xu,"Big community, small city" of ecological city analysis, master's thesis Shenyang University of Technology, 2013.

[4] Huinan Guo,, Zunmin Wu, Baolei Han, Chinese Learning City: from international to local change and reconstruction, Open Education Research, vol.6, pp. 112-118, 2015.

[5] Deyang Li, Zhaogang Sun, the evolution of cultural ecosystem, Journal of Wuhan University of Technology(Social Sciences Edition), vol.2, pp. 97-101,2003 . 
[6] Aiming Yang, The Pan Ecochain theory ecosystem of social-economic-natural, Science of Soil and Water Conservation, vol.1, pp. 93-96, 2005. 